\title{
Noncontact monitoring of heart rate responses to taste stimuli using a video camera
}

\author{
Masnani Bt Mohamed ${ }^{1,2}$, Makoto Yoshizawa ${ }^{3}$, Norihiro Sugita ${ }^{4}$, Shunsuke Yamaki ${ }^{3}$, Kei Ichiji ${ }^{5}$ \\ ${ }^{1}$ Graduate School of Biomedical Engineering, Tohoku University, Japan \\ ${ }^{2}$ Faculty of Electrical and Electronic Engineering, Universiti Tun Hussein Onn Malaysia, Malaysia \\ ${ }^{3}$ Cyberscience Center, Tohoku University, Japan \\ ${ }^{4}$ Graduate School of Engineering, Tohoku University, Japan \\ ${ }^{5}$ Graduate School of Medicine, Tohoku University, Japan
}

\begin{tabular}{l} 
Article Info \\
\hline Article history: \\
Received Aug 1, 2019 \\
Revised Oct 2, 2019 \\
Accepted Nov 6, 2019 \\
\hline
\end{tabular}

\section{Keywords:}

Basic taste

Electrocardiogram

Image plethysmogram

Noncontact measurement

Physiological signals

\begin{abstract}
Pleasant or unpleasant feeling stimulated by taste usually expressed to describe the acceptance or rejection of food and beverages intake by a human. Since it stimulates the emotional reactions, therefore it also induces other response such as heart rate variations. Traditional sensory tools used only subjective measurement such as self-report, to estimate the feeling of tasting. This method sometimes failed to show some differences between the pleasant and unpleasant type of feelings unconsciously. Previous unconscious measurement methods used the intrusive technique by placing some sensors in contact with the body, which may affect the results of sensory analysis. This study was conducted to avoid the effects of using contact sensors and validate the contact-less method of monitoring heart rate due to emotional changes by extracting plethysmographic signal from the green component of the video images. The videos were recorded while the subject responded to pleasant, unpleasant and neutral stimuli. The findings indicated that the heart rate was significantly related to taste stimuli that also reflected the subjective feelings. The unpleasant-taste influenced heart rate to increase more compared to pleasant-taste and neutral-taste. This proposed approach can be used to remotely detect the feeling/emotion that not overtly express through facial expression, speech or gestures.
\end{abstract}

Copyright $(2020$ Institute of Advanced Engineering and Science. All rights reserved.

\section{Corresponding Author:}

Masnani Bt Mohamed,

Graduate School of Biomedical Engineering,

Tohoku University, Sendai,

Miyagi 980-8579, Japan.

Email: masnani@uthm.edu.my

\section{INTRODUCTION}

Food and beverages appearance, aroma and taste give so much influence on a human to decide whether they like or not to consume the items. The sense of taste is essential for human to select good contents for ingestion by distinguishing so-called "basic" tastes; sweet, umami, sour, salty and bitter [1]. Recent studies have demonstrated the connection between food stimuli and emotions [2]. Each taste can induce pleasure or/and displeasure when various physiological responses occur associated with the gustatory stimuli. The change in the physiological signals can be used to relate the type of emotions evoked by taste [3-5]. Traditionally, the sensory evaluation commonly based on conscious and verbal/self-reported responses from participants to estimate their feeling of tasting and assess food acceptability by using only questionnaires or hedonic scales. By contrast, physiological responses analysis would instead be more appropriate to evaluate the sensational aspects due to taste perception, and it turns out to be a faster unconscious rating method [3]. Recently, heart rate has been used as one of the most common parameters to assess physiological responses associated with 
consumption and visual assessment of food and beverages [6-8]. Immediate and automatic responses with none or very little time for thinking is a better decision-making process for selecting the most appropriate item.

Most of the recent studies used the unconscious responses of the human body by integrating heart rate measurement with noninvasive multimodal techniques, such as brainwaves, body temperature, respiration levels, skin conductance, and facial expressions to assess the emotions elicited by taste. However, these methods are intrusive, discomfort and challenging to apply since they required electrodes to be attached to the body, such as on the hands, chest, and scalp [9-11]. These might not present accurate results, due to the possibility of affecting sensory, behavior and physiological responses such as increasing of arousal caused by the stress/anxiety of having sensors attached to the bodies and being monitored [7-8]. Therefore, the use of contact method is less appropriate because it could produce bias results in sensory analysis of food and beverages since the subjects will be aware of the measurements that may influence their heart rate and other physiological responses [12]. It also can cause skin irritation for a long monitoring session and a risk of losing contact when the subjects are moving. Recent advancements of measuring physiological signals by contactless or remote methods have progressively developed since it is noninvasive, nonintrusive, simple and lowcost for measuring multiple physiological parameters [13-17]. The monitoring of heart rate using video analysis is an alternative method developed based on the photoplethysmogram (PPG) concept. Instead of using red and infrared lights, the PPG signals can also be measured by extracting the green component, which is absorbed by hemoglobin and oxyhemoglobin at a relatively shallow part of the skin [18]. The changes in blood volume will be evident at green wavelengths, and thus the blood flow can be measured in the skin surface especially in the cheeks and forehead, where the highest blood flow usually happened. This blood flow event can be recorded by using a video camera and further analyzed using signal processing technique to convert into the heart rate value [19].

Most of the standard heart rate estimation using video camera only measures the heart rate without emotional influences. They did not emphasize on the evaluation changes of the heart rate as an emotional measure or the effect of stimuli on autonomic arousal. The most well-studied emotional expression methods by noncontact technique only based on facial expressions, eye gaze, body gestures, and speech signals. Thus, this study was aimed to investigate and validate the noncontact method in measuring the heart rate changes due to emotional reactions caused by pleasant and unpleasant feeling by capturing the light reflected from the subject's face known as the image photoplethysmogram (iPPG). This study is meant to be used as a tool to identify and understand the emotional state of a person, even the emotion is not overtly expressed.

\section{RESEARCH METHOD}

\subsection{Experiment Materials}

The subjects consisted of ten individual healthy university students aged around 21-23 years old. However, the data recorded from the two subjects cannot be analyzed due to the technical problems. All subjects were free from medications and illness, non-smokers, no allergy for the drinks offered and not suffering from smell or taste deficiencies. They were requested to refrain from eating or drinking for at least 1 hour prior to the experiment session. The stimuli proposed for evoking flavor sensations were chosen based on an investigation performed by previous researchers $[3,5]$. They defined the chocolate cream/sucrose as a sweet flavor and rated it as the most pleasant. On the other hand, the lemon homogenate/citric acid was considered as a sour flavor and rated as strongly unpleasant. Meanwhile, the flavorless item or mineral water rated as neither pleasant nor unpleasant and used as a control stimulus. Based on the information, the taste samples used in this experiment were including the solutions of milk chocolate (sweet flavor), lemon juice (sour flavor) and mineral water (flavorless). Specifically, the sweet solution was used for evoking pleasant sensation whereas sour solution for evoking unpleasant sensation. On the other hand, natural mineral water was served as the control stimulus and also used for mouth cleaning.

The experiments were conducted in an environment-controlled laboratory in such that the temperature and humidity were set at ambient values. There were three tasting sessions; sweet-taste (pleasant), sour-taste (unpleasant) and water-taste (neutral). The subjects individually tasted each solution, approximately $20 \mathrm{ml}$ in a session lasted about $50 \mathrm{~s}-60 \mathrm{~s}$. The heart rate at rest was monitored for $30 \mathrm{~s}$ before the presentation of taste solutions and it was stored separately. At the start, the subjects took a sip of the solution and left it in their mouth for about $10 \mathrm{~s}$ for a complete impregnation over the entire oral area before they swallowed it. They had to restrain the facial reactions and maintain to look directly at the video camera. They also placed their chin and forehead on a headrest to minimize the head movement. In the last part of each experiment, the subjects were requested to do self-report on the degree of liking or disliking of stimulus using hedonic scales. They identified the taste of the solutions and scored their feeling: 7, Very pleasant; 6, Moderately pleasant; 5, Slightly present; 4, Neither pleasant nor unpleasant; 3, Slightly unpleasant; 2, Moderately unpleasant and 1, Very unpleasant. The experimental procedures were repeated identically for the other type of solutions. 


\subsection{Recording System and Data Acquisition}

This contact-less method used only a CMOS video camera with a white LED light source placed approximately $70 \mathrm{~cm}$ in front of the subjects. They were facing a video camera (TGX02c; Baumer Co., Ltd) during the recording sessions. All videos were recorded in color image at 120 frames per seconds (fps) with 440x400 pixel resolution. The reference heart rate was monitored simultaneously throughout the experiment using the 3-lead ECG electrodes connected to a BIOPAC System (MP150) with AcqKnowledge (v.3.9.1) software. All the recorded video files and ECG signals were further analyzed using MATLAB (ver. R2017b). The electrocardiographic R-waves were detected offline and the intervals between heartbeats were converted into heart rate in beats per minute (bpm).

\subsection{Heart Rate Estimation Method}

Figure 1 demonstrates the main idea to estimate the heart rate from a video image. The plethysmographic signal was obtained by averaging the pixel values of the selected region of interest (ROI) in each video frame. After that, the signal was further analyzed using a signal processing technique to eliminate noises before estimating the heart rate.

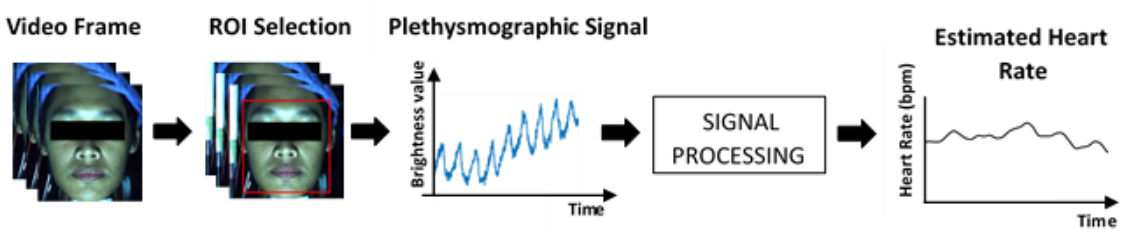

Figure 1. The basic process of heart rate estimation using video image

The color intensity-based method for obtaining heart rate from a face image is presented in Figure 2. A box ROI in Figure 2(a) was manually selected in the area that consists of many facial skin pixels after smoothing the whole image using the median filter. The image photoplethysmographic (iPPG) signal in Figure 2(b) was obtained by averaging the brightness of the green component at each pixel values inside the ROI.

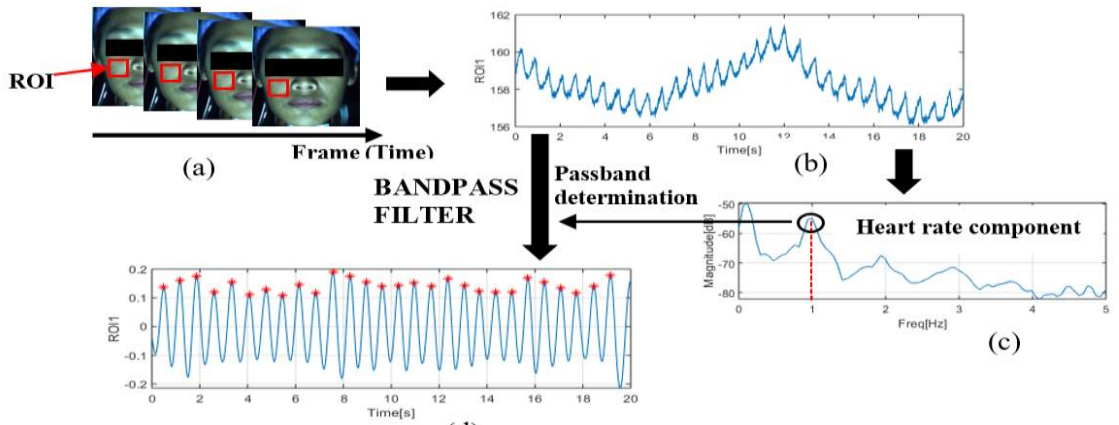

(d)

Figure 2. Heart rate estimation method

For the heart rate extraction, some filter processing was performed to remove the noise due to the variation of lighting, body movement, breathing, and camera adjustment. A linear trend and abrupt changes were also removed from the pulse wave signal that was obtained as the time series of the average value. The heart rate computation can be grouped into time-domain analysis and frequency-domain analysis [20]. The signal was transformed into the frequency domain using the Welch method. By using the power spectral density estimation, the dominant frequency with the largest amplitude as in Figure 2(c) is defined as the average heart rate. For the time domain processing, a Butterworth filter with the pass-band of $0.7-2.0 \mathrm{~Hz}$ was applied to remove noise components except for cardiac frequency components. It fits the heart rate variability between 40-120 bpm for healthy people [21]. The signal's maximum point interval that is known as a beat-to-beat interval (BBI) in Figure 2(d) can be used to estimate the instantaneous heart rate by multiplying the reciprocal of BBI by 60 . 


\section{RESULTS AND ANALYSIS}

\subsection{Image Photoplethysmogram (iPPG) and Electrocardiogram (ECG)}

The heart rate variability affected by taste stimuli is demonstrated in Figure 3. It is an example of a subject's heart rate when responding to a pleasant taste. It is meant to compare the data taken via a video camera (iPPG) with the ECG module. Both techniques have almost the same results except during the facial movements, which is around $14 \mathrm{~s}-21 \mathrm{~s}$, where facial heart rate fluctuated and different from ECG values. This condition was happened due to the movement of the mouth area, including facial reacting while swallowing the solution. The data during this period are not reliable and not accurate. Therefore, the data during stimulation from facial video image that contaminated with facial movements/reactions should be discarded. The results from iPPG had no significant differences $(P>0.05)$ with ECG before and after stimulation, as displayed in Figure 3(b). This, therefore, proved that the iPPG is an acceptable technique to measure the heart rate reacted to taste stimuli provided no facial reactions are involved.

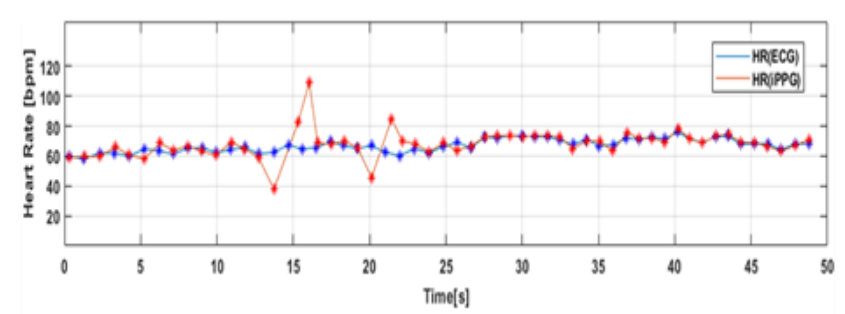

(a)

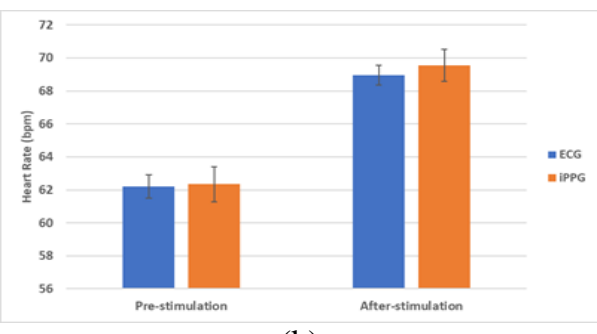

(b)

Figure 3. (a) Heart rate measurement in response to pleasant taste using both ECG (Blue) and video camera (Red), (b) Average heart rate before and after stimulation

\subsection{Reference Heart Rate}

The reference heart rate was measured with a 3-lead ECG module in order to compare and validate the results measured using the noncontact technique. The heart rate variations and the percentage of the heart rate increase/decrease in response to pleasant (sweet), unpleasant (sour) and neutral (water) stimuli are shown in Figure 4. The pleasant and unpleasant stimuli significantly increased heart rate responses immediately after the presentation of the taste solutions, whereas the neutral stimulus decreased the heart rate concerning the prestimulus value.

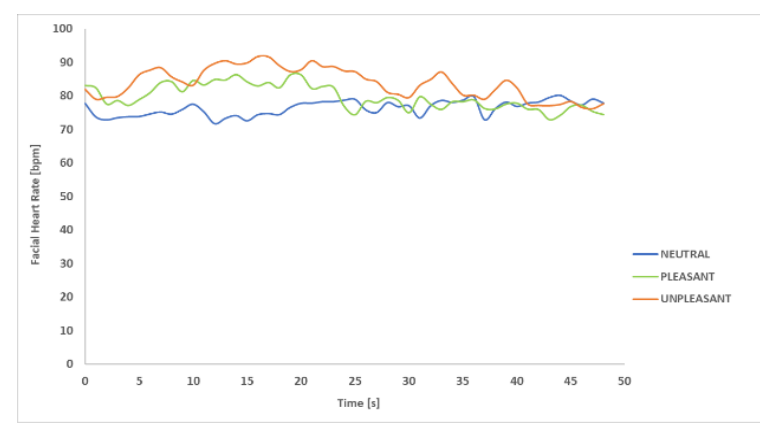

(a)

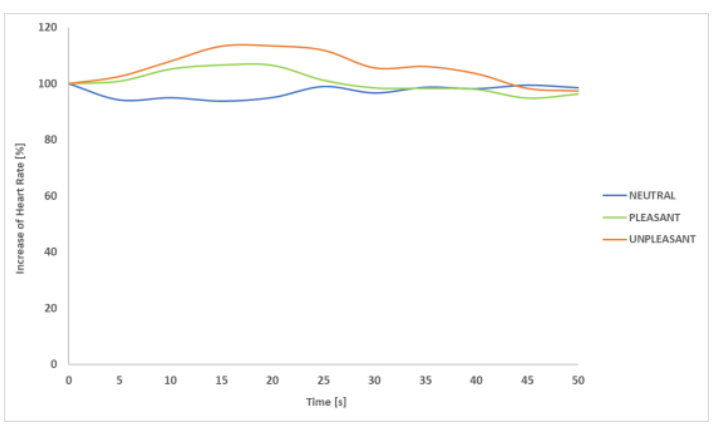

(b)

Figure 4. ECG measurement, (a) Heart rate variations, (b) Heart rate changes (\%)

The mean heart rate at rest measurement using ECG was $79.2 \pm 1.6$ (mean $\pm \mathrm{SD}$ ) for the $30 \mathrm{~s}$ before the presentation of the taste solutions. This value is considered as $100 \%$. The increase/decrease of the mean heart rate for every $5 \mathrm{~s}$ after the presentation of the solutions are shown in Figure 4(b). Basically, the heart rate started to increase during the stimulation period (in the first $10 \mathrm{~s}$ ) while consuming pleasant and unpleasanttaste until $10 \mathrm{~s}$ later they reached the peak heart rate. The maximum increases in heart rate induced by pleasant and unpleasant-taste were $6.6 \%$ and $13.5 \%$ respectively. However, mineral water (neutral-taste) lowered down the heart rate by $6.2 \%$ after $10 \mathrm{~s}$ of presentation of the solution. These heart rate changes were compared with 
the mean level of the heart rate at rest. The sour solution was rated as unpleasant taste and it induced the acceleration of heart rate immediately after $5 \mathrm{~s}$ of presentation of the solution. Meanwhile, the sweet solution also increased the heart rate but slightly lower than the sour solution. By contrast, the neutral solution decreased the heart rate immediately before it returned to pre-stimulus level (after $30 \mathrm{~s}$ ). Overall, the average increases after-stimulation for neutral, pleasant and unpleasant-taste were $-3.9 \%, 4.8 \%$, and $13 \%$ respectively. Even though the effect of mineral water was inverted from the effect of sour and sweet stimuli, but it showed a significant change $(P<0.05, \mathrm{~F}>$ Fcrit) before and after consuming it. The ANOVA test for all taste stimuli also proved that the mean heart rate after-stimulation had significant differences with pre-stimulation heart rate $(P<0.05, \mathrm{~F}>\mathrm{Fcrit})$. The results are consistent with the previous finding where the heart rate increases induced by the water (neutral) and sucrose (sweet) were significantly lower than those induced by citric acid (sour) [3].

\subsection{Facial Heart Rate/Image Photoplethysmogram (iPPG)}

In the case of monitoring heart rate using a video camera, the measurement on the forehead ROI also shows the changes after experiencing each taste stimulus compared to pre-stimulus condition (Figure 5). The facial heart rate or image Photoplethysmograms (iPPG) showed that the pleasant and unpleasant stimuli significantly increased the heart rate responses after the presentation of the taste solutions whereas, neutral stimulus immediately decreased the heart rate after consuming it. The mean heart rate at rest measurement using video camera was $78.1 \pm 2.3$ (mean $\pm \mathrm{SD}$ ). This value is considered as $100 \%$. The increases/decreases of the mean heart rate for every $5 \mathrm{~s}$ after the presentation of the solutions are shown in Figure $5(\mathrm{~b})$. The heart rate changes were compared with the mean level of the heart rate at rest. The results show the similar trends of heart rate variations as measured using ECG where they also started to accelerate during the stimulation period (in the first $10 \mathrm{~s}$ ) for pleasant and unpleasant-taste until they reached the maximum heart rate of $7 \%$ and $14.2 \%$. Whereby, neutral-taste lowered down the heart rate by $4.2 \%$ after $10 \mathrm{~s}$ of presentation of the solution. Overall, the average increases after-stimulation for neutral, pleasant and unpleasant-taste were $-2.3 \%, 5.9 \%$, and $12.6 \%$ respectively. All differences were considered significant at a level of $P<0.05$ before and after-stimulation.

The accuracy estimation of the average heart rate measured in different ROI concerning the root means square error and the correlation coefficient is displayed in Figure 6. The selected ROIs including right cheek, left cheek and forehead. The heart rate measurement seems to be more accurate in the forehead area, especially for the case of measuring the effect of an unpleasant solution. This because of the forehead area was less affected while the subject was swallowing the solutions. The correlation coefficient in Figure 6(b) also displayed the relationship between heart rate measured using ECG and video camera in the forehead that are significantly related for all type of solutions.

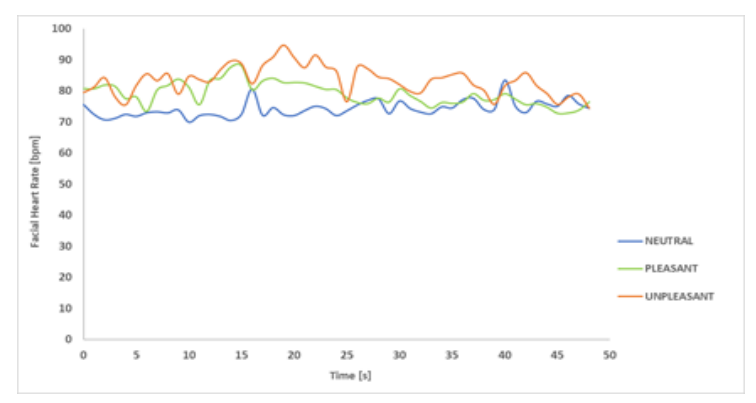

(a)

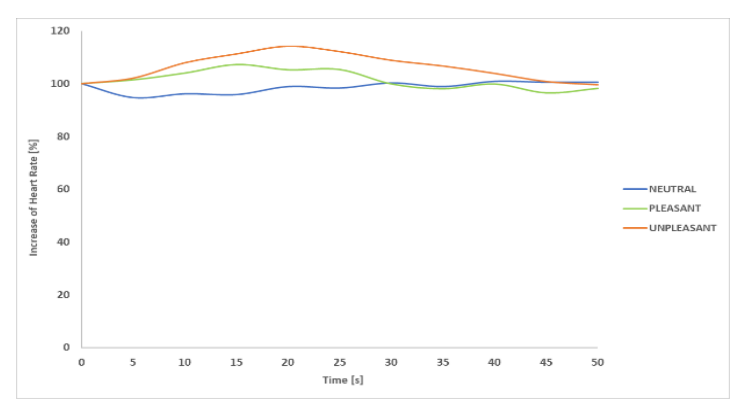

(b)

Figure 5. iPPG measurement, (a) Heart rate variations, (b) Heart rate changes (\%)

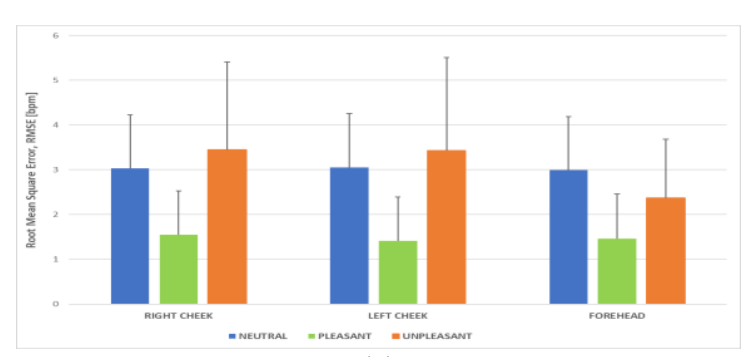

(a)

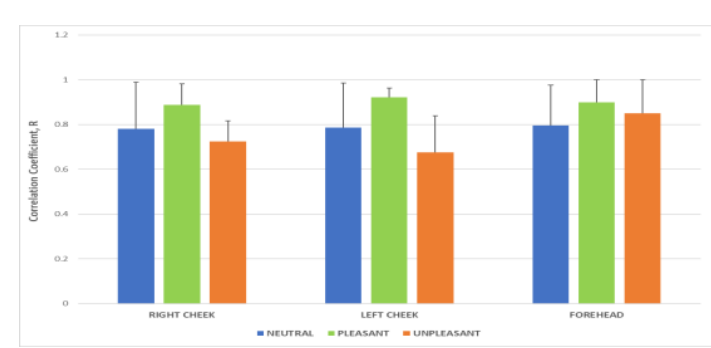

(b)

Figure 6. Accuracy estimation in different ROI, (a) RMSE, (b) Correlation coefficient 


\subsection{Discussion}

The purpose of this study was to characterize each basic taste by heart rate responses, which objectively reflect the subject's emotional reactivity. The variations in the heart rate were induced through the autonomic nervous system [22]. This study found that the unpleasant sour taste provoked a negative feeling hence, activated stronger heart rate response compared to pleasant sweet taste that provoked a positive feeling. This may due to pleasant sweet taste is becoming a familiar and usual taste hence, can be considered that the human has become "habituated" to sweet taste [23]. The ANOVA test for all three types of taste also gave the values of $\mathrm{F}>\mathrm{F}$ crit and $P<0.05$, which determined the significant differences between all heart rates before and after stimulation. The percentage of change in the heart rate triggered by unpleasant-taste solution increased by $13 \%$ (ECG) and $12.6 \%$ (iPPG) right after the stimulation. Meanwhile, the percentage of change in the heart rate triggered by pleasant-taste solution increased by $4.8 \%$ (ECG) and $5.9 \%$ (iPPG). By contrast, the percentage of change in heart rate triggered by neutral-taste decreased by $-3.9 \%$ (ECG) and $-2.3 \%$ (iPPG) after the subjects consuming the solution. That means the sour solution significantly affect the feeling compared to the sweet and water solution used in this experiment. This accordance with the previous study reported that the rat neonatal heart rate responses increased after stimulation with lemon [24-25]. Not to mention, this study also showed that the forehead was accurately measured the heart rate compared to the left cheek and right cheek.

Overall, the magnitude of heart rate responses could reflect the acceptance (lower the heart rate activation associated with sweet and mineral water) or rejection (stronger heart rate activation associated with sour) situation. Based on the results, the magnitude of the heart rate response could reflect the pleasant or unpleasant feeling that can be used to relate with acceptance or rejection of any food, drinks or medications consumption as well. When consuming bad tastes, humans try to evoke the rejection signal by demonstrating a higher heart rate. They tend to react negatively in certain emotions like sadness, anger, surprise, disgust and scared. Whereas, when consuming good tastes, they demonstrated positive emotions of happy or neutral that does not change the heart rate higher than during negative feelings.

\section{CONCLUSION}

In conclusion, this study has validated the practicability of implementation of a contact-less technique using only a video camera to assess the sensory analysis related to taste. This technique replaced the traditional physiological sensors with a camera, which will increase the usability of the detecting system and will overcome the complication of using contact sensors. The results showed that the significant correlation exists between taste stimuli and heart rate that reflected very subjective feelings. The unpleasant-taste induced the negative feeling thus exhibited a stronger heart rate response with higher amplitude compared to others. Nevertheless, the neutral taste was induced neutral feeling and displayed very weak amplitude. Besides, the response shows the increasing pattern as well for pleasure feeling due to the excitement, but lower than the displeasure feeling. This study produced a technique that has the advantage to sense the feeling in an unconscious situation. This technique can be useful in the detection of emotional/feeling state of person even if he/she does not overtly express his/her emotion through gestures, speech or facial expressions. In the future, this technique can be integrated with other modalities, such as facial expressions and gestures in order to enhance the emotion detection method. The nonrigid facial movements/expressions could affect the result of facial heart rate. Therefore, this work will be continued by improvising a method to overcome the nonrigid movements, especially in the ROI area. Not to mention, the distinction between initial and subsequent responses discovered by this technique using facial heart rate may offer a new valuable perspective for sensory and consumers' eating behavior research. Due to the inexpensiveness and the fact that this method is relatively easy to implement even using only a smartphone camera, this method has the potential to become widespread.

\section{ACKNOWLEDGEMENTS}

This research was supported by the COI Stream Project funded by the Japanese Ministry of Education, Science, Sports, and Culture. The authors also would like to thank Universiti Tun Hussein Onn Malaysia (UTHM), Ministry of Higher Education Malaysia (MOHE), the laboratory members and the volunteers for the contributions, assistance, and supports.

\section{REFERENCES}

[1] N. Chaudhari and S. D. Roper, “The Cell Biology of Taste,” J. Cell Biol., vol. 190, no. 3, pp. 285-296, 2010.

[2] D. V. Smith and M. B. Vogt, "The Neural Code and Integrative Processes of Taste," in Tasting and Smelling, Handbook of Perception and Cognition, 2nd ed., G. K. Beauchamp and L. Bartoshuk, Eds. San Diego, California: Academic Press, 1997, pp. 25-76. 
[3] S. Rousmans, O. Robin, A. Dittmar, and E. Vernet-Maury, "Autonomic Nervous System Responses Associated with Primary Tastes,” Chem. Senses, vol. 25, pp. 709-718, 2000.

[4] M. Bensafi, et al., "Autonomic Nervous System Responses to Odours: The Role of Pleasantness and Arousal," Chem. Senses, vol. 27, pp. 703-709, 2002.

[5] P. Muroni and R. Crnjar, "Emotional Responses to Pleasant and Unpleasant Oral Flavour Stimuli," Chemosens. Percept., vol. 4, no. 3, pp. 65-71, 2011.

[6] S. D. Kreibig, "Autonomic Nervous System Activity in Emotion : A Review," Biol. Psychol., vol. 84, no. 3, pp. 14$41,2010$.

[7] D. D. Torrico, et al., "Images and Chocolate Stimuli Affect Physiological and Affective Responses of Consumers : A Cross-Cultural Study," Food Qual. Prefer., vol. 65, pp. 60-71, 2018.

[8] C. Gonzalez Viejo, et al., "Integration of Non-invasive Biometrics with Sensory Analysis Techniques to Assess Acceptability of Beer by Consumers," Physiol. Behav., pp. 1-9, 2018.

[9] R. A. De Wijk, et al., "ANS Responses and Facial Expressions Differentiate Between the Taste of Commercial Breakfast Drinks," PLoS One, vol. 9, no. 4, pp. 1-9, 2014.

[10] R. A. De Wijk, et al., "Autonomic Nervous System Responses on and Facial Expressions to the Sight, Smell, and Taste of Liked and Disliked Foods," Food Qual. Prefer., vol. 26, pp. 196-203, 2012.

[11] C. Brown and A. B. Randolph, "The Story of Taste: Using EEGs and Self-reports to Understand Consumer Choice," Kennesaw J. Undergrad. Res., vol. 2, no. 1, 2012.

[12] C. G. Viejo and S. Fuentes, "Non-contact Heart Rate and Blood Pressure Estimations from Video Analysis and Machine Learning Modelling Applied to Food Sensory Responses : A Case Study for Chocolate," Sensors, vo. 18, no. 1802, pp. 1-18, 2018.

[13] M. A. Hassan et al., "Heart rate estimation using facial video: A review," Biomed. Signal Process. Control, vol. 38, pp. 346-360, 2017.

[14] M.-Z. Poh, D. J. McDuff, and R. W. Picard, "Advancements in Noncontact, Multiparameter Physiological Measurements Using a Webcam," IEEE Trans. Biomed. Eng., vol. 58, no. 1, pp. 7-11, 2011.

[15] M.-Z. Poh, D. J. McDuff, and R. W. Picard, "Non-contact, Automated Cardiac Pulse Measurements Using Video Imaging and Blind Source Separation.," Opt. Express, vol. 18, no. 10, pp. 10762-10774, 2010.

[16] H. E. Tasli, A. Gudi, and M. Den Uyl, "Remote PPG Based Vital Sign Measurement Using Adaptive Facial Regions," 2014 IEEE Int. Conf. Image Process. ICIP 2014, pp. 1410-1414, 2014.

[17] N. Sugita, et al., "Contactless Technique for Measuring Blood-Pressure Variability from One Region in Video Plethysmography," J. Med. Biol. Eng., pp. 1-10, 2018.

[18] W. Verkruysse, L. O. Svaasand, and J. S. Nelson, "Remote plethysmographic imaging using ambient light," Opt. Express, vol. 16, no. 26, pp. 21434-21445, 2008.

[19] N. Ibrahim, et al., "Non-contact Heart Rate Monitoring Analysis from Various Distances with Different Face Regions," Int. J. of Electrical and Electronic Eng., vol. 7, no.6, pp. 3030-3036, 2017.

[20] C. Wang, T. Pun, and G. Chanel, "A Comparative Survey of Methods for Remote Heart Rate Detection from Frontal Face Videos,” Front. Bioeng. Biotechnol., vol. 6, no. 33, pp. 1-14, 2018.

[21] N. Sugita, et al., "Techniques for Estimating Blood Pressure Variation Using Video Images," Proc. 37th Annu. Conf. IEEE Eng. Med. Biol. Soc., pp. 4218-4221, 2015.

[22] T. Horio, "Effects of Various Taste Stimuli on Heart Rate in Humans," Chem. Senses, vol. 25, no. 2, pp. 149-153, 2000.

[23] A. Leterme, L. Brun, A. Dittmar, and O. Robin, "Autonomic Nervous System Responses to Sweet Taste: Evidence for Habituation Rather Than Pleasure," Physiol. Behav., vol. 93, pp. 994-999, 2008.

[24] W. P. Smotherman and S. R. Robinson, "Heart Rate Response of the Rat Fetus and Neonate to a Chemosensory Stimulus," Physiol. Behav., vol. 50, no. 33, pp. 47-52, 1991.

[25] T. Horio and Y. Kawamura, "Salivary Secretion Induced by Umami Taste," J. Oral Biosci., vol. 31, pp. 107-111, 1989.

\section{BIOGRAPHIES OF AUTHORS}

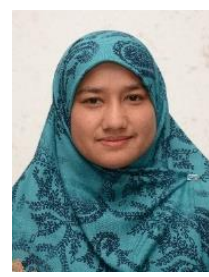

Masnani Bt Mohamed received the B.Eng. Degree in Electrical from Universiti Teknologi MARA, Shah Alam, Malaysia in 2003. Three years later (2006) she received her M.Eng. degree in Electrical (Electronics and Telecommunications) from Universiti Teknologi Malaysia, Johor, Malaysia. She is currently a lecturer at the Department of Electronic Engineering, Faculty of Electrical and Electronic Engineering (FKEE), UTHM, Johor, Malaysia. She was a Head of Medical Electronic Lab from 2010 until 2015 at Faculty of Electrical and Electronic Engineering, UTHM. She is a member of Board of Engineers Malaysia from 2014 until the present. She was a member of Microelectronics \& Nanotechnology Shamsuddin Research Centre and Biomedical Engineering Modelling and Simulation (BioMEMS) focus group at FKEE, UTHM. Her research interests include the heart sound, brainwaves and electrocardiogram analysis. Currently, she focuses on developing a noncontact measurement of physiological signals. 


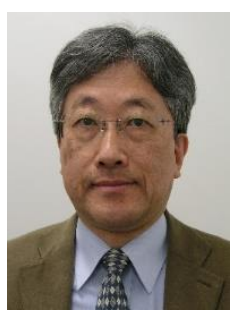

Yoshizawa Makoto received the B.S., M.S. and Ph.D. degrees in Electrical and Communication Engineering from Tohoku University in 1978, 1980 and 1983, respectively. He was a Research Associate from 1983 to 1991 in the same department. Since 1991 to 1994, he was an Associate Professor at Toyohashi University of Technology, Toyohashi, Japan. In 1994, he returned to Tohoku University. He became a Visiting Scientist, Research Institute of Medicine, Johns Hopkins University, Baltimore and Baylor College of Medicine, Houston, U.S.A. in 1999. Since 2001, he has been a Professor in the Research Division on Advanced Information Technology, Information Synergy Center (currently, Cyberscience Center), Tohoku University. He engages in the application of virtual reality to medicine, intelligent control of artificial hearts, assessment of the effects of visual stimulation on humans and tele-healthcare. He was a member of AdCom of IEEE EMBS from 2009 to 2011. He has been a member of Editorial Committee and a councilor of Journal of the Japanese Society for Medical and Biological Engineering.

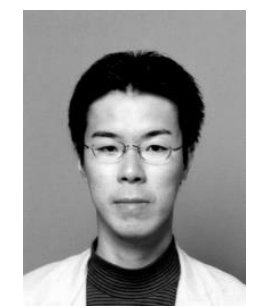

Norihiro Sugita received the B.S., M.S. and Ph.D. degrees in engineering from Tohoku University, Sendai, Japan, in 1998, 2001 and 2004, respectively. He was a COE Research Fellow from 2004 to 2006 and an Assistant Professor from 2006 to 2010 in the Department of Electrical and Communication Engineering, Graduate School of Engineering, Tohoku University. He is currently an Associate Professor in the Department of Management Science and Technology, Graduate School of Engineering, Tohoku University. His research interests include the application of virtual reality to medicine, assessment of the effects of visual stimulation on humans and tele-healthcare. $\mathrm{He}$ is a member of the Japanese Telemedicine and Telecare Association and the Society of Instrument and Control Engineers of Japan.

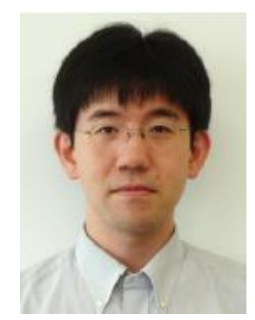

Shunsuke Yamaki received the B.E., M.E., and D.E. degrees in electronic engineering from Tohoku University, Sendai, Japan, in 2004, 2006, and 2008, respectively. He is currently an Assistant Professor in Research and Development Divisions, Cyberscience Center at Tohoku University. His research interests include digital filter design and linear system theory. He received the President Prize of Tohoku University in 2006, the Best Research Award in the 4th StudentOrganizing International Mini-Conference on Information Electronics System (SOIM-COE06) in 2007, the Young Excellent Author Award of the 19th IEICE Workshop on Circuits and Systems in Karuizawa in 2007, Excellent Presentation Award in The Society of Instrument and Control Engineers, Tohoku Chapter in 2009, The 7th Noguchi Excellent Research Award in Information Processing Society of Japan, Tohoku Branch in 2012, Best Paper Award in The 3rd IEEE International Conference on Network Infrastructure and Digital Content (IC-NIDC) in 2012, M. Ishida Foundation Excellent Research Award in 2012, the SIP Young Excellent Author Award of the 27th IEICE SIP Symposium in 2013, and the Young Engineer Award from the IEICE in 2014. $\mathrm{He}$ is a member of the IEEE, the Society of Instrument and Control Engineers of Japan, and the Information Processing Society of Japan. He is an IEEE Senior Member.

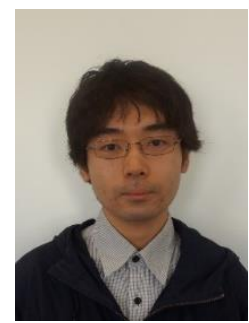

Kei Ichiji graduated from Tokyo Metropolitan College of Industrial Technology, Japan and received the B.Eng. Degree from National Institution for Academic Degrees, Japan in 2009. He received the M.Eng. and Ph.D. degrees in engineering from Tohoku University, Japan, in 2011 and 2014 respectively. He is currently an Assistant Professor at Tohoku University Graduate School of Medicine. He has engaged in the application of signal processing for intelligent control of the radiation therapy machine. He is a member of the Society of Instrument and Control Engineers, the American Association of Physicists in Medicine, the IEEE CIS and the IEEE EMBS. 